\title{
Calidad de vida de cuidadores familiares de personas con cáncer que reciben atención de cuidados paliativos
}

\author{
Quality of life of family caregivers of people with cancer in palliative care \\ Qualidade de vida dos cuidadores familiares de pessoas com câncer em cuidados paliativos
}

Como citar este artículo:

Arredondo Holgín E, Arias-Rojas M, Carreño Moreno S. Quality of life of family caregivers of people with cancer in palliative care. Rev Esc Enferm USP. 2021;55:e03740. https://doi.org/10.1590/S1980-220X2020015103740

\author{
Edith Arredondo Holgín' \\ Mauricio Arias-Rojas ${ }^{1}$ \\ Sonia Carreño Moreno ${ }^{2}$ \\ ${ }^{1}$ Universidad de Antioquia, Facultad \\ de Enfermería, Grupo de investigación \\ GIPECS, Medellín, Antioquia, Colombia. \\ ${ }^{2}$ Universidad Nacional de Colombia, Facultad \\ de Enfermería, Grupo de Investigación \\ Cuidado de Enfermería al Paciente Crónico, \\ Bogotá, Cundinamarca, Colombia.
}

\begin{abstract}
Objective: To describe quality of life and related factors in a sample of Colombian caregivers of people with cancer in palliative care. Method: A correlational, descriptive and cross-sectional study conducted with 208 family caregivers of people with cancer in outpatient palliative care in Medellín, Colombia. The Quality of Life in Life-Threatening Illness - Family Caregiver Version (QOLLTI-F) instrument was used. Results: Quality of life obtained scores between 116.36 and 122.35 (95\%CI). The dimensions with the lowest scores were Patient condition (2.4-3.2, 95\%CI), Caregiver's own state (36.2-39.9, 95\% CI) and Environment $(14.4-15.7,95 \% \mathrm{CI})$. The patient's and caregiver's ages and the Karnofsky index presented a positive correlation, from weak to moderate and significant with the caregiver's quality of life. The daily hours devoted to care presented a weak correlation, negative and significant, with the overall quality of life and with the Caregiver's own state dimension. Conclusion: It is necessary to develop interventions to improve quality of life in caregivers of people with advanced cancer in the palliative phase, considering the importance of relief actions and the management of the patient's functional dependence.
\end{abstract}

DESCRIPTORS

Quality of Life; Caregivers; Neoplasm; Palliative Care.
Autor correspondiente:

Mauricio Arias-Rojas

Universidad de Antioquia, Facultad de

Enfermería, Grupo de investigación GIPECS

Calle 64 \#53-09, oficina 323

050035 - Medellín, Antioquia, Colombia

Emauricio.arias@udea.edu.co
Recibido: 28/04/2020

Aprobado: 03/12/2020 


\section{INTRODUCCIÓN}

Las enfermedades crónicas son una realidad creciente en el mundo y el cáncer aporta de forma significativa a la carga global de estas. Acorde con la Organización Mundial de la Salud (OMS), para el 2016, el cáncer cobró la vida de nueve millones de personas, lo que representó el 22\% de la carga por mortalidad atribuible a la enfermedad crónica ${ }^{(1)}$.

Sumado a la problemática de la mortalidad por cáncer, se encuentra el hecho de que, no hay integración suficiente del cuidado paliativo en los sistemas de provisión de servicios en salud a nivel mundial. Acorde con PAL-LIFE ${ }^{(2)}, 35$ millones de personas viven anualmente con condiciones crónicas susceptibles de cuidado paliativo, de las cuales fallecen 25 millones, sin recibir, en muchas ocasiones, el tipo de cuidado que requieren. Un claro ejemplo de dicha desintegración, es el caso colombiano, en donde hay deficiencias estructurales, de acceso, de formación de recurso humano y culturales en torno al cuidado paliativo ${ }^{(3)}$; por ejemplo, existen menos de 0,5 servicios de cuidado paliativos por millón de habitantes, existen 17 unidades de atención para cerca de 47 millones de habitantes, los servicios se concentran en grandes ciudades, se consume una cifra baja de $6,7 \mathrm{mg}$ de opioide por año por persona y solo existen 3 programas en de cuidados paliativos documentados.

La realidad de la problemática asociada al cuidado paliativo no es solo de la persona que lo requiere, si no de su cuidador familiar, que en ocasiones permanece invisible y sus necesidades desatendidas, situación que afecta su calidad de vida. Los cuidadores familiares de personas en cuidado paliativo reportan más bajos niveles de calidad de vida, que la población en general y que los cuidadores de personas con cáncer que tienen intención curativa del tratamiento. Un estudio reportó diferencias marcadas en las dimensiones de salud mental y rol emocional, entre la población general y los cuidadores familiares después de la muerte del paciente; además deterioro significativo en la salud general y en la dimensión física ${ }^{(4)}$. De igual manera, otro estudio ${ }^{(5)}$, reportó peores resultados en las dimensiones funcionamiento físico, rol físico, salud general, vitalidad, funcionamiento social, rol emocional y salud mental, que la población general, es decir, con excepción de la dimensión dolor físico, en general la calidad del vida del cuidador de la persona con cáncer en cuidados paliativos, es significativamente más baja, que la calidad de vida de la población general.

Se han reportado niveles más bajos de calidad de vida en cuidadores de personas con cáncer gástrico en cuidado paliativo, en comparación con la media de la población general; además, factores como la edad joven en cuidadores y pacientes, se relacionaron con mejor salud física; se encuentra además que cuidadores con edad avanzada, con mejor salud percibida, que no son cónyuges del paciente y a quienes su paciente no había recibido ninguna cirugía y no estaban hospitalizados, reportaron mejores niveles de calidad de vida en el área de salud mental ${ }^{(6)}$.

En cuanto a la calidad de vida relacionada con la salud, un estudio en Alemania mostró que los cuidadores familiares de personas con cáncer en fase paliativa, presentaron niveles más bajos comparados con la población general, además, las mujeres reportaron peores niveles de calidad de vida en el ámbito emocional que los hombres; la baja calidad de vida en las mujeres puede estar relacionado con la alta carga emocional que desarrollan ${ }^{(7)}$. En contraste, un estudio en Noruega, reportó bajos niveles de calidad de vida en cuidadores familiares de personas con cáncer en fase paliativa tardía, con menores niveles en hombres que en mujeres del componente mental; aunque la literatura reporta mayor impacto emocional y mental en mujeres que en hombres, este estudio no se confirmó esa hipótesis; una explicación alternativa son las diferencias culturales ${ }^{(8)}$.

El estudio de la calidad de vida en cuidadores de personas con cáncer en cuidados paliativos se ha concentrado en describir y comparar los niveles de calidad de vida con los de la población general, además de explicar factores influyentes como se describió anteriormente. Considerando primero que situaciones de orden cultural pueden ser mediadoras de la calidad de vida y no solamente las relacionadas con el cuidado de la persona con cáncer en cuidados paliativos, y segundo que los sistemas de salud de muchos países de latinoamerica aún no integran el cuidado paliativo dentro de la provisión de los servicios de salud, lo que lleva a la necesidad de realizar descripciones en contextos como el latinoamericano. Al respecto, en la revisión a la fecha solo se encontró un estudio realizado con cuidadores de personas con cáncer en cuidado paliativo en Chile, donde se reportaron niveles de calidad de vida similares a los de estudios realizados con cuidadores de personas con cáncer hospitalizadas; además, reportaron peores resultados en la salud mental que en la salud física y niveles significativamente menores en la vitalidad, el rol emocional y la salud mental en mujeres que en hombres ${ }^{(9)}$.

Dados los pocos estudios que hay en latinoamérica frente a la descripción de la calidad de vida del cuidador familiar del paciente con cáncer en cuidados paliativos, este estudio planeteó como objetivo describir la calidad de vida y factores relacionados en una muestra de cuidadores familiares colombianos de personas con cáncer en cuidados paliativos.

\section{MÉTODO}

\section{Diseño DEL ESTUdio}

Estudio de diseño cuantitativo, descriptivo correlacional, de corte transversal.

\section{PoblaCión Y ESCENARIO}

Este estudio fue desarrollado en un servicio de pacientes ambulatorios de un hospital de tercer nivel de atención, especializado en la atención de personas con cáncer en la ciudad de Medellín-Colombia.

\section{CRITERIOS DE SELECCIÓN}

Para este estudio se tuvo en cuenta dentro de los criterios de inclusión que el participante fuera el cuidador familiar principal del paciente con cáncer en estadio IV que estuviera recibiendo atención de cuidados paliativos ambulatorios, que fuera mayor de 18 años. Se excluyeron cuidadores que fueran 
contratados. Se realizó muestreo por conveniencia, con la participación de 208 cuidadores familiares.

\section{ReCOlECCIÓN DE DATOS}

Para colectar los datos de los participantes del estudio, se aplicó la encuesta de caracterización de la diada versión cuidados paliativos (GCPC-UN-D-CP), diseñada por el Grupo de Investigación Cuidado de Enfermería al Paciente Crónico de la Universidad Nacional de Colombia. La encuesta identificas las características sociodemográficas del cuidador y el paciente, variables relacionadas con la provisión de cuidados, tales como el tiempo que lleva como cuidador, si es el único cuidador y número de horas diarias dedicadas a cuidar. Esta encuesta se ha utilizados en varios estudios en Latinoamérica y cuenta con características adecuadas de validez facial y de contenido ${ }^{(10)}$.

Para medir la calidad de vida, se usó el instrumento denominado Calidad de Vida en Enfermedades de Amenazan la Vida, versión cuidador familiar en español (QOLLTI-F) ${ }^{(11)}$. El instrumento fue creado con base en un estudio cualitativo en que se indagó con los cuidadores acerca de los aspectos que son importantes para su calidad de vida. Mediante análisis factorial se confirmó la validez del instrumento, con una varianza explicada de 53\%; además se reportó una confiabilidad test re-test de 0,77 a 0,80. Este instrumento, ha sido documentado como único, dado que mide la calidad de vida, informada por el cuidador familiar e incluye la condición del paciente; lo que confirma la relación estrecha entre los dos. Además se ha documentado que es especial, dado que se derivó de un estudio cualitativo en el que se indagó a los cuidadores sobre los aspectos importante para su calidad de vida, en lugar de, centrarse en los cambios o cargas derivadas del hecho de cuidar. Esta escala, presentó adecuadas propiedades psicométricas para el contexto colombiano. En un estudio no publicado por los autores, en cuidadores de personas con cáncer en cuidado paliativo, se confirmó su estructura factorial de 6 dimensiones, con un modelo que explicó el 70\% de la varianza. Además, se documentó una consistencia interna alfa de cronbach de 0,82 y una estabilidad test retest de 0,879 . En la versión colombiana el instrumento tiene 16 ítems, con las dimensiones de medio ambiente ( 2 ítems), estado del paciente ( 1 ítem), estado del cuidador (6 ítems), perspectiva del cuidador (3 ítems), calidad del cuidado (2 ítems) y relaciones (2 ítems). La escala cuenta con opciones de puntaje en una escala de 0 a 10 donde a mayor puntaje, mayor nivel de calidad de vida, excepto por los ítems 3, 4 y 15 que están invertidos.
La recolección de la información se llevó a cabo durante el segundo semestre de 2019. Los cuidadores fueron contactados, a través del programa de cuidado paliativo ambulatorio de la institución. Los investigadores y dos auxiliares de investigación previamente entrenados, invitaron a participar a los cuidadores, revisaron que cumplieran los criterios de inclusión y explicaron, los objetivos del estudio para posteriormente realizar la firma del consentimiento informado escrito. La participación de los cuidadores fue voluntaria. Una vez obtenido el consentimiento, se procedió a administrar la hoja de caracterización sociodemográfica y la escala de calidad de vida. Este procedimiento se realizó en un lugar reservado y tranquilo en la institución, la aplicación del instrumento duró en promedio de 10 a 15 minutos.

\section{ANÁlISIS Y TRATAMIENTO DE LOS DATOS}

El análisis se adelantó con el paquete estadístico SPSS versión 24, licenciado para la Universidad de Antioquia, Colombia. Para la descripción de las variables demográficas y de cuidado de los participantes, se acudió a estadística descriptiva, con el uso de distribuciones de frecuencias relativas, medidas de tendencia central y dispersión. Para la descripción de las variables cuantitativas continuas como edad, tiempo que lleva como cuidador, horas diarias dedicadas a cuidar y calidad de vida, se usaron medidas de tendencia central, dispersión e intervalos de confianza del 95\%. Para explorar los factores asociados a la calidad de vida del cuidador, se usaron coeficientes de correlación de Pearson, previo cumplimiento de requisitos de normalidad e inversión de los puntajes de las respuestas a los ítems 3, 4 y 15.

\section{Aspéctos ÉTICOS}

Este estudio fue aprobado por el comité de ética e investigación de la institución en que fue conducido (aval $n^{\circ}$ 11-2018). Todos los participantes firmaron el consentimiento informado escrito.

\section{RESULTADOS}

\section{Características de los PARTICIPANTES}

Se incluyeron 208 cuidadores familiares principalmente de género femenino (79\%), con una edad media de 49,72 años, con nivel educativo de secundaria (36\%) que cuidaban a la persona con cáncer desde el momento de su diagnóstico (90\%). Las características sociodemográficas y perfil del cuidador se presenta en la Tabla 1.

Tabla 1 - Características de los cuidadores familiares participantes - Medellín, Colombia, 2019.

\begin{tabular}{|c|c|c|c|}
\hline Variable & & Recuento & Porcentaje \\
\hline \multirow{2}{*}{ Género } & Femenino & 164 & $79 \%$ \\
\hline & Masculino & 44 & $21 \%$ \\
\hline \multirow{2}{*}{ Grado máximo de escolaridad } & Posgrado & 19 & $9 \%$ \\
\hline & Primaria & 33 & $16 \%$ \\
\hline
\end{tabular}




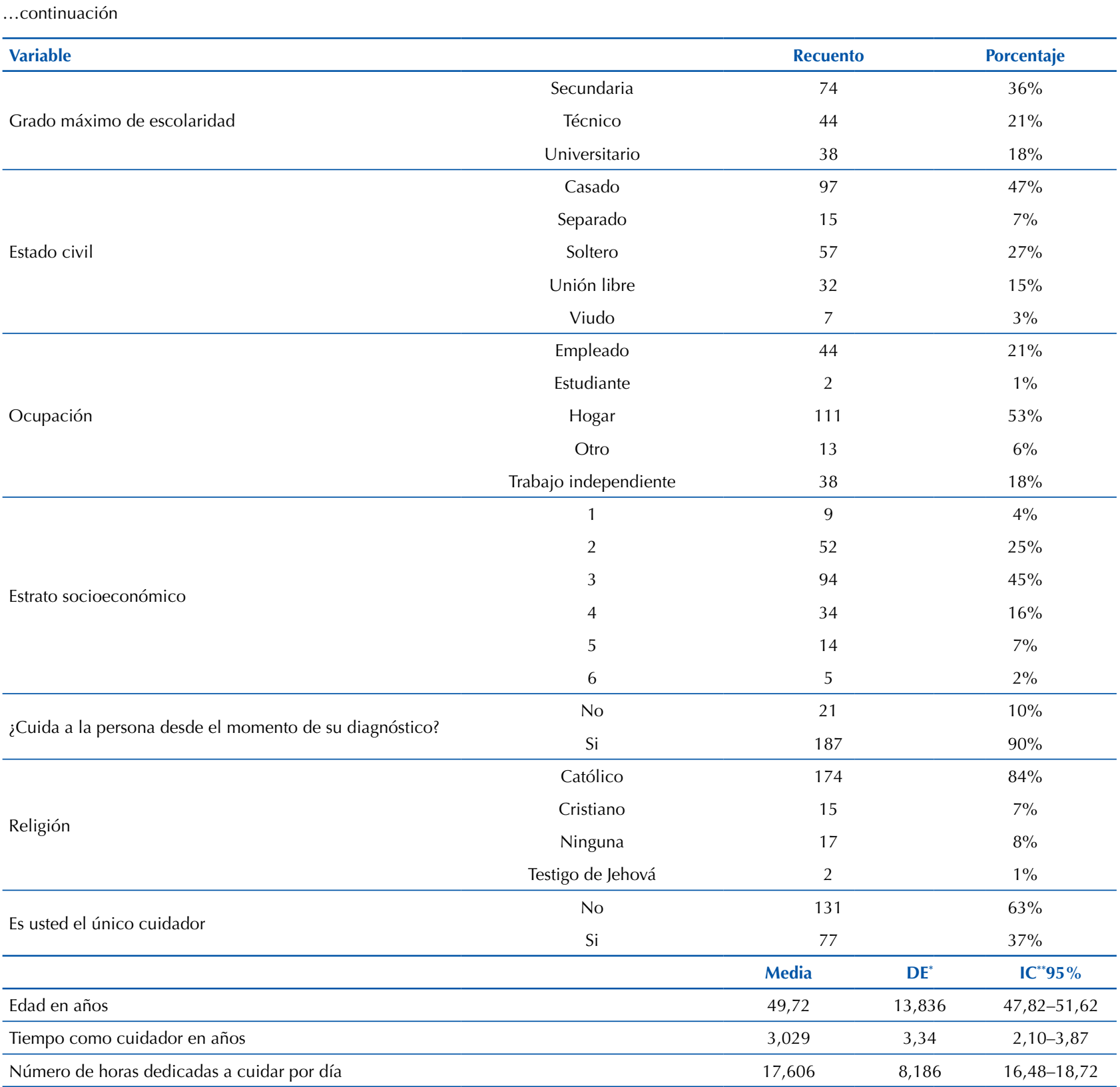

*Desviación estándar; **Intervalo de confianza

En la Tabla 2 se presentan las características de los pacientes en cuidados paliativos que cuidaban los participantes del estudio.

\section{DESCRIPCIÓN DE LA CALIDAD DE VIDA}

La Tabla 3 presenta la descripción de la calidad de vida en enfermedades que amenazan la vida, informada por los cuidadores participantes del estudio.

Tabla 2 - Características de los pacientes en cuidados paliativos - Medellín, Colombia, 2019.

\begin{tabular}{|c|c|c|c|}
\hline \multicolumn{2}{|c|}{ Variable/Tipo de estadístico } & \multirow{2}{*}{$\frac{\text { Recuento/Descriptivos }}{3}$} & \multirow{2}{*}{$\frac{\text { Porcentaje }}{1 \%}$} \\
\hline & Cáncer hueso & & \\
\hline & Cáncer de pulmón & 23 & $11 \%$ \\
\hline \multirow[t]{3}{*}{ Diagnóstico } & Cáncer de ovario & 44 & $21 \%$ \\
\hline & Cáncer de mama & 28 & $13 \%$ \\
\hline & Cáncer gastrointestinal & 32 & $15 \%$ \\
\hline
\end{tabular}




\begin{tabular}{|c|c|c|c|c|}
\hline \multicolumn{2}{|c|}{ Variable/Tipo de estadístico } & \multicolumn{2}{|c|}{ Recuento/Descriptivos } & Porcentaje \\
\hline \multirow{8}{*}{ Diagnóstico } & Cáncer renal & \multicolumn{2}{|c|}{3} & $1 \%$ \\
\hline & Cáncer de cabeza y cuello & \multicolumn{2}{|c|}{38} & $18 \%$ \\
\hline & Cáncer endocrino & \multicolumn{2}{|c|}{9} & $4 \%$ \\
\hline & Cáncer cerebral & \multicolumn{2}{|c|}{9} & $4 \%$ \\
\hline & Cáncer de próstata & \multicolumn{2}{|c|}{4} & $2 \%$ \\
\hline & Cáncer uterino & \multicolumn{2}{|c|}{3} & $1 \%$ \\
\hline & Linfoma & \multicolumn{2}{|c|}{8} & $4 \%$ \\
\hline & Mieloma & \multicolumn{2}{|c|}{4} & $2 \%$ \\
\hline \multirow{3}{*}{ Género } & Femenino & \multicolumn{2}{|c|}{137} & $66 \%$ \\
\hline & Masculino & \multicolumn{2}{|c|}{71} & $34 \%$ \\
\hline & & Media & $\mathrm{DE}^{*}$ & IC95\% \\
\hline Edad en años & & 62,55 & 16,21 & $60,33-64,76$ \\
\hline Escala de Kar & & 62,64 & 6,61 & $61,74-63,55$ \\
\hline
\end{tabular}

*Desviación estándar

Tabla 3 - Calidad de vida de los cuidadores familiares - Medellín, Colombia, 2019.

\begin{tabular}{lccc}
\hline \multirow{2}{*}{ Calidad de vida } & & Estadísticos & IC95\% \\
\cline { 2 - 4 } QOLLTI-F total & Media & Desviación estándar & $116,36-122,35$ \\
Medio ambiente & 119,36 & 21,904 & $14,48-15,75$ \\
Estado del paciente & 15,12 & 4,633 & $2,40-3,28$ \\
Estado del cuidador & 2,84 & 3,214 & $36,22-39,94$ \\
Perspectiva del cuidador & 38,08 & 13,601 & $26,77-27,81$ \\
Calidad del cuidado & 27,29 & 3,795 & $18,28-18,99$ \\
Relaciones & 18,63 & 2,630 & $16,95-17,86$ \\
\hline
\end{tabular}

\section{FACTORES ASOCIADOS A LA CALIDAD DE VIDA DEL CUIDADOR}

El puntaje total de calidad de vida en enfermedades que amenazan la vida, se asoció con la edad en años del cuidador $(\mathrm{r}=.307)$ y número de horas diarias dedicadas a cuidar $(\mathrm{r}=-.155)$. La dimensión medio ambiente, se asoció con la edad en años del cuidador $(\mathrm{r}=.244)$; la dimensión estado del cuidador, se asoció con la edad del paciente $(\mathrm{r}=.162)$, edad del cuidador $(\mathrm{r}=.240)$ y horas diarias dedicadas a cuidar $(\mathrm{r}=-.181)$; la dimensión perspectiva del cuidador, se asoció con el puntaje de la escala Karnofsky $(\mathrm{r}=.143)$ y la edad del cuidador $(\mathrm{r}=.228)$; finalmente, la dimensión relaciones, se asoció con la edad del cuidador $(r=.225)$. Todas las asociaciones fueron significativas con una $\mathrm{p}<0,05$.

\section{DISCUSIÓN}

Frente a las características de las personas con cáncer avanzado en cuidado paliativo, este estudio reporta una escala Karnofsky media de 62,64 puntos, variados diagnósticos de cáncer y media de edad de 62,55 años. Estas características son similares a las reportadas en un metanálisis de ocho estudios, conducidos en escenario ambulatorio y domiciliario, que comprendieron una muestra total de 2.385 pacientes, en donde se reportó un ECOG entre cero y dos, variados tipos de cáncer avanzado y una edad media de 64,30 años $^{(12)}$.

Las características de los cuidadores familiares principales, expresan un perfil con predominancia femenina, con edad media de 49,72 años, con escolaridad en niveles de bachillerato y técnico, con unión conyugal, de ocupación hogar, con mayor distribución de frecuencia en niveles socioeconómicos bajos y medios, que cuidan a la persona desde el momento del diagnóstico, con responsabilidades de cuidado compartidas con otros miembros de la familia y con alta dedicación al cuidado en meses y horas diarias. Las características descritas son similares a las reportadas en un estudio en Colombia ${ }^{(13)}$, a excepción de la dedicación como únicos cuidadores, la cual fue menor en el presente estudio. La razón por la cual la dedicación al cuidado no es exclusiva de un único cuidador en este estudio, puede deberse a que el estudio se condujo en una ciudad intermedia y con tradiciones culturales de cuidado que recáen en varios miembros de las familias, en donde el apoyo al cuidador principal es mayor. De igual forma, 
las características de los cuidadores familiares reportadas por este estudio, son similares a las reportadas en una muestra de cuidadores italianos ${ }^{(14)}$.

Los niveles de calidad de vida en los cuidadores de personas con cáncer avanzado en cuidados paliativos en este estudio, se alejaron de los niveles óptimos; el puntaje total de calidad de vida se ubicó entre 116,36 y 122,35 puntos de 160 posibles. Los resultados de este estudio, difieren en gran medida de los reportados otros estudios realizados en India ${ }^{(15)}$, en el que se halló una media de calidad de vida de 63,36 (DE: 15,73); en Alemania ${ }^{(16)}$ en un estudio de intervención con cuidadores de adultos con cáncer en fase paliativa, en el que la línea base de calidad de vida fue de 61 (DE: 12) y otro estudio también en Jordania ${ }^{(17)}$ con cuidadores familiares de pacientes con cáncer avanzado, que reportó una media de calidad de vida de 82,80 puntos. Los tres estudios referidos encontrados muestran una media muy inferior a la reportada por este estudio.

Por otra parte, las dimensiones que presentaron puntajes más bajos en este estudio, fueron la de estado del paciente, estado del cuidador y medio ambiente, hallazgos similares a los reportados por en un estudio realizado en Republica Checa, en donde las dimensiones con peores resultados fueron las de estado del cuidador y condiciones del paciente ${ }^{(18)}$. Resulta interesante que si bien, en este estudio el cuidador no era el único que cuidaba al paciente, se presentaron unos valores bajos frente al estado del cuidador; frente a esto, la evidencia ha documentado que cuidar a través de largos periodos de tiempo genera un desgaste en la salud física y psicológica del cuidador que impacta en su calidad de vida ${ }^{(19)}$.

En cuanto a los factores asociados a la calidad de vida la mayoría de estudios, han señalado al género como un factor asociado, en particular, se ha documentado que las mujeres que cuidan a personas con cáncer avanzado en fase paliativa, tienen calificaciones más bajas de calidad de vida que los hombres ${ }^{(7)}$; este hallazgo no fue ratificado en el presente estudio. Una explicación verosímil, tiene que ver con la distribución por género de la muestra de este estudio, la que fue en mayor proporción compuesta por mujeres.

La edad del paciente, edad del cuidador e índice Karnofsky, presentaron una correlación positiva, de débil a moderada y estadísticamente significativa con la calidad de vida del cuidador o alguna de sus dimensiones; dicho de otra manera, entre más edad del paciente y del cuidador, y mejor estatus funcional, mejores puntajes de calidad de vida. $\mathrm{Al}$ respecto, un estudio reportó que cuidadores familiares jóvenes informaban mejores niveles de calidad de vida y que cuidadores de pacientes con pobre estatus funcional, presentaban niveles más bajos de calidad de vida ${ }^{(18)}$. Por otra parte, los hallazgos de otro estudio en cuidadores brasile$\tilde{n} \mathrm{~s}^{(9)}$, controvierten lo hallado en este estudio frente a la edad como factor relacionado con la calidad de vida, puesto que en diez modelos de regresión, no identificaron la edad como un factor predictivo de la calidad de vida del cuidador, ni de sus dimensiones. En este sentido, se ratifica aquí, que un buen estatus funcional del paciente, influye de forma positiva en la calidad de vida; sin embargo, frente al hallazgo acerca de la edad del paciente y cuidador, se requiere mayor investigación que explique la naturaleza de la relación. Una explicación alternativa es el estilo de afrontamiento de las personas mayores, la cual se ha documentado con tendencia a la resignación ${ }^{(20)}$, además del desarrollo de factores de resiliencia que tiene la presente generación de adultos mayores en Medellín-Colombia, que han estado expuesto a décadas de conflicto $\operatorname{armado}^{(21)}$.

Las horas diarias dedicadas a cuidar, presentaron una correlación débil, negativa y estadísticamente significativa, con la calidad de vida total y la dimensión estado del cuidador. Al respecto, un estudio ${ }^{(9)}$ reportó que el tiempo de cuidado diario como predictor de la calidad de vida del cuidador de la persona con cáncer en cuidados paliativos, en el dominio de rol físico, al igual que otros autores ${ }^{(19)}$ quienes reportaron niveles más bajos de salud mental y bienestar emocional en cuidadores que gastaban más tiempo al día brindando cuidados.

Este estudio reconoce varias limitaciones. Primero, la selección de los participantes no se dio de forma aleatoria así que queda futuros estudios deben determinar si la distribución de los cuidadores y los resultados en este estudio, se dan de igual manera en una muestra seleccionada de forma aleatoria. Segundo, los participantes de este estudio fueron captados de un programa de atención ambulatoria de cuidados paliativos, en donde el estado de salud del paciente y sus necesidades pueden estar mayormente estables frente al contexto de pacientes que están hospitalizados y su impacto en el cuidador.

Frente a las fortalezas del estudio se reconoce que el tamaño de la muestra de 208 cuidadores es mayor a lo reportado en otros estudios en el área. Así mismo, la descripción detallada de la muestra de este estudio servirá para hacer comparaciones con futuros estudios que evalúen la calidad de vida de los cuidadores.

\section{CONCLUSIÓN}

La calidad de vida reportada en este estudio osciló entre 116,36 y 122,35 (IC95\%). Las dimensiones con las puntuaciones más bajas fueron las de estados del paciente $(2,4-3,2$, IC95\%), estado del cuidador (36,2-39,9, IC95\%) y medio ambiente (14,4-15,7, IC95\%). La edad del paciente, edad del cuidador e índice Karnofsky, presentaron una correlación positiva, de débil a moderada y estadísticamente significativa con la calidad de vida del cuidador o alguna de sus dimensiones. Las horas diarias dedicadas a cuidar, presentaron una correlación débil, negativa y estadísticamente significativa, con la calidad de vida total y la dimensión estado del cuidador.

Se requiere desarrollar intervenciones para mejorar la calidad de vida de los cuidadores de personas con cáncer avanzado en fase paliativa, considerando la importancia de acciones de relevo y manejo de la dependencia funcional del paciente. Se requiere explorar el rol de la edad como factor influyente en la calidad de vida del cuidador. 


\section{RESUMEN}

Objetivo: Describir la calidad de vida y factores relacionados, en una muestra de cuidadores colombianos de personas con cáncer en cuidados paliativos. Método: Estudio descriptivo correlacional de corte transversal, con 208 cuidadores familiares de personas con cáncer en cuidados paliativos ambulatorios en Medellín-Colombia. Se usó el instrumento de calidad de vida en enfermedades que amenazan la vida - versión cuidador familiar (QOLLTI-F). Resultados: La calidad de vida puntuó entre 116,36 y 122,35 (IC95\%). Las dimensiones con las puntuaciones más bajas fueron del estado del paciente (2,4-3,2, IC95\%), estado del cuidador (36,2-39,9, IC 95\%) y medio ambiente (14,4-15,7, IC95\%). La edad del paciente, edad del cuidador e índice Karnofsky, presentaron una correlación positiva, de débil a moderada y significativa con la calidad de vida del cuidador. Las horas diarias dedicadas a cuidar, presentaron una correlación débil, negativa y significativa con la calidad de vida total y la dimensión estado del cuidador. Conclusión: Se requiere desarrollar intervenciones para mejorar la calidad de vida de los cuidadores de personas con cáncer avanzado en fase paliativa, considerando la importancia de acciones de relevo y manejo de la dependencia funcional del paciente.

\section{DESCRIPTORES}

Calidad de Vida; Cuidadores; Neoplasias; Cuidados Paliativos.

\section{RESUMO}

Objetivo: Descrever a qualidade de vida e fatores relacionados em uma amostra de cuidadores colombianos de pessoas com câncer em cuidados paliativos. Método: Estudo descritivo transversal correlacional com 208 cuidadores familiares de pessoas com câncer em cuidados paliativos ambulatoriais em Medellín-Colômbia. Foi utilizado o instrumento de qualidade de vida para doenças com risco de vida - versão cuidador familiar (QOLLTI-F). Resultados: A qualidade de vida foi pontuada entre 116,36 e 122,35 (IC95\%) As dimensões com as pontuações mais baixas foram status do paciente (2,4-3,2; IC95\%), status do cuidador (36,2-39,9; IC95\%) e ambiente (14,4-15,7; IC95\%). A idade do paciente, a idade do cuidador e o índice de Karnofsky apresentaram correlação positiva, fraca a moderada, e significativa com a qualidade de vida do cuidador. As horas diárias dedicadas ao cuidado apresentaram correlação fraca, negativa e significativa com a qualidade de vida total e a dimensão estado do cuidador. Conclusão: É necessário desenvolver intervenções para melhorar a qualidade de vida dos cuidadores de pessoas com câncer avançado na fase paliativa, considerando a importância das ações de alternância entre cuidadores e o manejo da dependência funcional do paciente.

\section{DESCRITORES}

Qualidade de Vida; Cuidadores; Neoplasias; Cuidados Paliativos.

\section{REFERENCIAS}

1. World Health Organization. Noncommunicable diseases: country profiles 2018 [Internet]. Geneva: World Health Organization; 2018 [cited 2020 Mar 6]. Available from: http://apps.who.int/iris/bitstream/handle/10665/274512/9789241514620-eng.pdf?ua=1

2. Centeno C, Sitte T, Lima L, Alsirafy S, Bruera E, Callaway M, et al. Documento de posición oficial sobre la promoción global de cuidados paliativos: recomendaciones del grupo internacional asesor PAL-LIFE de la Academia Pontificia de la Vida, Ciudad del Vaticano. J Palliat Med. 2018;21(10):1398-407. https://doi.org/10.1089/jpm.2018.0387

3. Pastrana T, De Lima L, Pons JJ, Centeno C. Atlas de cuidados paliativos de Latinoamérica: edición cartográfica 2013 [Internet]. Houston: International Association for Hospice and Palliative Care; 2013 [cited 2020 Mar 6]. Available from: https://cuidadospaliativos.org/ uploads/2014/3/Atlas\%20Latinoamerica\%20-\%20edicion\%20cartografica.pdf

4. Götze H, Brähler E, Gansera L, Schnabel A, Gottschalk-Fleischer A, Köhler N. Anxiety, depression and quality of life in family caregivers of palliative cancer patients during home care and after the patient's death. Eur J Cancer Care. 2018;27(2):e12606. https://doi.org/10.1111/ ecc. 12606

5. Ullrich A, Ascherfeld L, Marx G, Bokemeyer C, Bergelt C, Oechsle K. Quality of life, psychological burden, needs, and satisfaction during specialized inpatient palliative care in family caregivers of advanced cancer patients. BMC Palliat Care. 2017;16(1):31. https://doi. org/10.1186/s12904-017-0206-z

6. Morishita M, Kamibeppu K. Quality of life and satisfaction with care among family caregivers of patients with recurrent or metastasized digestive cancer requiring palliative care. Support Care Cancer. 2014;22(10):2687-96. https://doi.org/10.1007/s00520-014-2259-3

7. Choi S, Seo J. Analysis of caregiver burden in palliative care: an integrated review. Nurs Forum. 2019;54(2):280-90. https://doi. org/10.1111/nuf.12328

8. Tan JY, Molassiotis A, Lloyd-Williams M, Yorke J. Burden, emotional distress and quality of life among informal caregivers of lung cancer patients: an exploratory study. Eur J Cancer Care. 2018;27(1):e12691. https://doi.org/10.1111/ecc.12691

9. Toffoletto MC, Reynaldos-Grandón KL. [Social determinants of health, family overload and quality of life in family caregivers of cancer patients receiving palliative care]. Rev Salud Publica (Bogota). 2019;21(2):154-60. Spanish. https://doi.org/10.15446/rsap.v21n2.76845

10. Chaparro-Diaz L, Sánchez B, Carrillo-Gonzalez GM. Encuesta de caracterización del cuidado de la diada cuidadorfamiliar: persona con enfermedad crónica. Rev Cienc Cuid. 2014;11(2):31-45. https://doi.org/10.22463/17949831.196

11. Cohen R, Leis AM, Kuhl D, Charbonneau C, Ritvo P, Ashbury FD. QOLLTI-F: measuring family carer quality of life. Palliat Med. 2006;20(8):755-67. https://doi.org/10.1177/0269216306072764

12. Fulton JJ, LeBlanc TW, Cutson TM, Porter Starr KN, Kamal A, Ramos K, et al. Integrated outpatient palliative care for patients with advanced cancer: A systematic review and meta-analysis. Palliat Med. 2019;33(2):123-34. https://doi.org/10.1177/0269216318812633

13. Arias-Rojas M, Carreño-Moreno S, Posada-López C. Uncertainty in illness in family caregivers of palliative care patients and associated factors. Rev Lat Am Enfermagem. 2019;27:e3200. https://doi.org/10.1590/1518-8345.3185.3200

14. Franchini L, Ercolani G, Ostan R, Raccichini M, Samolsky-Dekel A, Malerba MB, et al. Caregivers in home palliative care: gender psychological aspects, and patient's functional status as main predictors for their quality of life. Support Care Cancer. 2019;28(7):2327-35 https://doi.org/10.1007/s00520-019-05155-8 
15. Nayak MG, George A, Vidyasagar MS, Kamath A. Quality of life of family caregivers of patients with advanced cancer. J Nurs Heal Sci [Internet]. 2014 [cited 2020 Mar 9];3(2):70-5. Available from: https://pdfs.semanticscholar.org/9fd2/c89465c76b87912931004959ffc9ced64972.pdf

16. Groh G, Vyhnalek B, Feddersen B, Führer M, Borasio GD. Effectiveness of a specialized outpatient palliative care service as experienced by patients and caregivers. J Palliat Med. 2013;16(8):848-56. https://doi.org/10.1089/jpm.2012.0491

17. Bani Younis MK, Al-Rawashdeh A, Alnjadat RM. The effect of palliative care intervention program on the quality of life among Jordanian caregivers of cancer patients. Home Health Care Serv Q. 2019;38(4):286-96. https://doi.org/10.1080/01621424.2019.1661325

18. Bužgová R, Kozáková R, Sikorová L. Assessment of quality of life of family members of inpatients with end-stage disease. J Palliat Care. 2015;31(4):250-7. https://doi.org/10.1177/082585971503100407

19. Bakitas MA, Tosteson TD, Li Z, Lyons KD, Hull JG, Li Z, et al. Early versus delayed initiation of concurrent palliative oncology care: patient outcomes in the ENABLE III randomized controlled trial. J Clin Oncol. 2015;33(13):1438-45. https://doi.org/10.1200/JCO.2014.58.6362

20. Hernández R, Calderon C, Carmona-Bayonas A, Rodríguez Capote A, Jara C, Padilla Álvarez A, et al. Differences in coping strategies among young adults and the elderly with cancer. Psychogeriatrics. 2019;19(5):426-34. https://doi.org/10.1111/psyg.12420

21. Gaviria SL, Alarcón RD, Espinola M, Restrepo D, Lotero J, Berbesi DY, et al. Socio-demographic patterns of posttraumatic stress disorder in Medellin, Colombia and the context of lifetime trauma exposure. Disaster Health. 2016;3(4):139-50. https://doi.org/10.1080/216650 44.2016.1263086 